\title{
VGEF Mixed-Backbone Antisense Oligonucleotide GEM 220
}

National Cancer Institute

\section{Source}

National Cancer Institute. VGEFMixed-Backbone Antisense Oligonucleotide GEM 220.

NCl Thesaurus. Code C2016.

A mixed-backbone antisense oligonucleotide that is complementary to a pro-angiogenic vascular endothelial growth factor (VEGF) mRNA sequence. Because of its antiangiogenic properties, GEM 220 has been studied as a potential antineoplastic agent. (NCI04) 